\title{
Measurement and modelling of the elastic defection of novel metal syntactic foam composite sandwich structures in 3-point bending
}

D Cheneler and A R Kennedy*

Department of Engineering, Lancaster University, LA1 4YW, UK

*Corresponding author: a.kennedy3@lancaster.ac.uk

\section{Abstract}

This paper presents a process for creating syntactic metal foam sandwich structures which, for the first time, manufactures both the syntactic foam core and metal face sheets in a single step. Unlike previous research, the face sheets are made from the same material, are integral with the sandwich structure and are relatively thick compared to the core. Via comparison with experimental measurements in 3-point bending, it is shown that accurate prediction of the elastic deformation of these sandwich structures necessitates FE-based modelling, but that the methodology is simple. A reliance on predictions from established analytical models, which have proven themselves accurate for foam sandwiches with thin face sheets, leads to significant overestimations of the stiffness. Modelling, based on the FEA approach developed herein, shows that mass savings between 20 and $30 \%$ are achievable for these novel structures, produced using simple and low cost casting methods, but are dependent upon the deflection response required.

\section{Keywords}

Sandwich structures; syntactic foams, porous metals, 3 point bending

\section{Introduction}

Just as polymer composite sandwiches have shown their capability to deliver lightweight structures; materials comprised of an aluminium foam core, with aluminium face sheets, have the same exciting potential. These hybrid structures possess a unique combination of high specific 
strength and stiffness, resilience at high temperature and excellent energy, sound and vibration absorption. They offer a novel degree of design freedom for a wide diversity of potential products [1] and, as a result, there are numerous ongoing trials of prototypes in the defence, rail, aerospace, leisure and shipbuilding sectors [2].

Sandwich structures can be made by bonding solid face sheets to metal foams, but this comes with high costs, reduced stiffness and poor recyclability. Producing a solid surface in-process is preferred [3-5], but multiple costly processing steps and careful process control are still required to achieve complex and accurate products with acceptable reproducibility of structure and properties $[2,6]$.

Whilst issues of the level of process control required to achieve the reproducibility necessary for exacting applications remain; cost at the point of sale is still the prominent limitation on rendering metal foam sandwich structures mainstream in engineering applications $[2,6,7]$. To remedy this, aluminium foam sandwich structures need to be created using affordable and scalable manufacturing processes, which combine material production with component manufacture. Previous work [8] has demonstrated that a mould filled with large (>2 $\mathrm{mm})$, expanded glass particles (EGP), can be infiltrated to produce a syntactic metal foam (SMF) using a simple, low cost casting process. As the EGP defines the porosity and pore structure, increased reproducibility was achieved compared with traditional, highly stochastic metal foaming processes and good mechanical performance was observed, superior to foams with equivalent structures made by replication, albeit with increased density [8]. The low cost and resource-efficient nature of the recycled EGP product, coupled with the simplicity of manufacture and promising mechanical performance, indicate that there is scope for this novel approach to be developed for sandwichtype structures, which hold the greatest potential for practical light weighting solutions [1]. Syntactic metal foam sandwiches are a relatively new research area, with authors $[9,10]$ claiming first investigations into their mechanical performance in 2015. Whilst there have been numerous 
studies that have measured (reviewed in $[9,10])$ and modelled the deformation of traditional metal foam sandwich structures [11-15], the structural characteristics of the unique materials created and tested in this study are distinctly different from those previously described.

This paper presents a process for creating syntactic metal foam sandwich structures which, for the first time, manufactures both the syntactic foam core and metal face sheets in a single step. Unlike previous research, the face sheets are made from the same material, are integral with the sandwich structure and are relatively thick compared to the core $[9-12,15]$. The research in this paper quantifies the elastic deflection, in 3-point bending, for these novel structures, develops and validates a methodology for modelling their elastic behaviour and provides key insights into how mass savings in structures using these novel materials might be maximised.

\section{Experimental methods}

Expanded soda-lime glass particles (EGP) were used (supplied by OMYA) to create the porosity. The approximate chemical composition (in wt.\%) of these particles is $69-73 \% \mathrm{SiO}_{2}, 11.5-13.5 \% \mathrm{Na}_{2} \mathrm{O}$, 7.8-9.25\% $\mathrm{CaO}, 1-3 \% \mathrm{Al}_{2} \mathrm{O}_{3}, 1.99-2.5 \% \mathrm{MgO}, 0.99-1.2 \% \mathrm{~K}_{2} \mathrm{O}$ and $0.49-0.59 \% \mathrm{Fe}_{2} \mathrm{O}_{3}$. The ellipsoidal beads, with a closed surface, have an average density of $0.35 \mathrm{~g} / \mathrm{cc}[16]$ and were sieved between 2 and $3 \mathrm{~mm}$ in diameter. Their morphology is shown in Figure $1 \mathrm{a}$.

Reference samples were made from the core and face sheet materials in order to generate input data to create and validate models. For the foam core samples, EGP were poured into a vented metal mould containing a rectangular cavity 28 wide $\times 10 \mathrm{~mm}$ thick $\times 90 \mathrm{~mm}$ deep. A packing fraction in the range of $0.66-0.69$ was obtained by tamping after filling and achieved consistently by ensuring that a set mass of EGP was filled to the same height in the mould. Moulds were placed into a preheated muffle furnace at $300^{\circ} \mathrm{C}$ and then infiltrated under vacuum, using a PerCast Vacuum Casting machine, at a vacuum level of approximately 0.9 bar. The infiltrant was an LM6 grade aluminium alloy, sourced from Norton Aluminium, heated to $700^{\circ} \mathrm{C}$. The specification 
for the chemical composition for this alloy is $10-13 \mathrm{wt} . \% \mathrm{Si}$, with all other elements present below $0.6 w t . \%$ (for fuller detail of the composition specification see [17]). For more complete details on the manufacturing process, refer to $[8,18]$. Cast samples, $28.0 \mathrm{~mm} \times 10.0 \mathrm{~mm}$ in cross section, were milled to $80 \pm 0.1 \mathrm{~mm}$ in length. Al-Si alloy samples were cast in this same mould, milled to $80 \pm 0.1 \mathrm{~mm}$ in length and additionally milled to thicknesses of 4,6 and $8 \pm 0.05 \mathrm{~mm}$.

Sandwich components were made by infiltrating molten AI-Si alloy into and around rigid preforms of EGP that were located within a metal mould. Preforms were made by mixing $15 \mathrm{~g}$ of EPG with $4 \mathrm{~g}$ of sodium silicate binder. Coated beads were placed in the same ( $28 \mathrm{~mm}$ wide $\times 10 \mathrm{~mm}$ thick) mould as was used to make solid Al-Si alloy samples and were tamped manually to achieve similar packing fractions. The structures were "set" rigid by saturation with $\mathrm{CO}_{2}$ for $2-3$ minutes. Figure $1 \mathrm{~b}$ shows an image of a preform within the mould used to create the sandwich structures, located so it cannot move during the casting process. Gaps, $5 \mathrm{~mm}$ deep and $22 \mathrm{~mm}$ wide, on either side of the $10 \mathrm{~mm}$ thick core, provide space for metal to flow to create the face sheets for the sandwich structure. Casting was performed using the same method as described earlier, facilitating infiltration of molten Al into the preform and through the rest of the mould, creating the core and the face sheets in one operation. Figure 2 a shows an example of an as-cast sandwich component, where the EGP particles can be seen running through the centre of the casting. Sandwich samples were milled to widths of $20 \pm 0.1 \mathrm{~mm}$, removing material from the faces with exposed EGP, and to $80 \pm 0.1 \mathrm{~mm}$ in length. Milling was also performed on some of the Al face sheets to reduce their thickness to 4, 3 and $2 \pm 0.05 \mathrm{~mm}$. Whilst this doesn't fit to the objective of a "single step" process, for this preliminary investigation, it avoids the need for multiple different moulds to achieve the same outcome. An image of a sandwich structure with a $10 \mathrm{~mm}$ thick core and $2 \mathrm{~mm}$ thick face sheets, ready for testing, is shown in Figure $2 b$.

In all cases, the sample density was measured from the geometry and the mass. 3-point bending tests were performed at a constant displacement rate of $0.1 \mathrm{~mm} / \mathrm{s}$ using an Instron universal testing machine. The loading span was $64.0 \mathrm{~mm}$. A pre-calibrated linear-variable differential 
transformer was used to accurately measure displacement (with an effective resolution, based on the signal conditioning hardware, of less than $0.1 \mu \mathrm{m}$ ) and was deployed on the underside of the sample, below the central loading point. The machining and installation of a bespoke fixture for the LVDT ensured it was located to within $\pm 0.1 \mathrm{~mm}$ of this point. Its location is shown in a schematic of the testing setup in Figure 3. The elastic load-deflection behaviour was measured by averaging over 4 loading and unloading cycles.

Figure 3 shows the geometry for the FE model used to simulate 3 point bending of simple beam and sandwich structures, generated and run in ANSYS Workbench 19.0 (ANSYS Inc., USA) in the static structural mode. The geometry was created in DesignModeller 19.0 (ANSYS Inc., USA). The supports and loading edge were defined as $5 \mathrm{~mm}$ radius rigid cylinders with lengths the same as the beam width. The beam was defined using primitive blocks, with dimensions as defined below. All beams were meshed using quadratic hexahedral (SOLID186) elements with nominal dimensions of $1 \mathrm{~mm}$. The contact between the beam and the supports and the leading edge was defined as frictionless. A rigid-deformable contact pair (using TARGE170 and CONTA174 elements with the rigid cylindrical surfaces set as the target face), using an augmented Lagrange contact algorithm with detection at the Gauss integration points, was used at each point of contact between the beam and the cylinders. For sandwich structures, 3 layers were created, and defined as "bonded", meaning no sliding or separation between faces or edges was allowed. Again, an augmented Lagrange contact algorithm with detection at the Gauss integration points was used on the internal interfaces of the sandwich structure.

A fixed displacement constraint was applied to the surface of the supports, while the loading edge was constrained in all directions and rotations, except for the vertical direction in which a downwards force was applied. The beam was constrained in orthogonal directions at 3 corners to prevent rigid body movement, without adding any additional stress. The material properties were defined as isotropic elastic. A lack of stiffness data for the EGP, coupled with the poor ability for simple analytical models to predict the stiffness of "composites" containing high volume fractions 
of highly porous, weak fillers, dictated the Young's modulus for the EGP composite core to be determined by the 3 point bending testing procedure described earlier. This approach was also taken for the cast Al-Si alloy used to make the face sheets. The Poisson's ratio, for both materials, was assumed to be 0.33 .

The simulations were static structural and large deformation analysis was included. During simulations, a force was applied through the central loading edge and the deformation was determined on the underside of the sample, below the loading position (to coincide with the LVDT placement - which is shown in Figure 3). The force was applied as a ramp over 10 sub steps. The global matrix was symmetric and sparse and a full Newton-Raphson algorithm was used in the solution.

\section{Results and discussion}

\section{Simple beams and model validation}

Figure 4 shows typical load-displacement plots for AI-Si (Al) and syntactic foam structures with a width of $28 \mathrm{~mm}$. The LVDT data show good linearity, even over small displacements, giving confidence to the measurement method. The reproducibility of measurement was high, with a scatter, defined by the standard deviation for the 4 testing cycles, of less than $2 \%$. The gradients for the samples are also displayed on the plot. As expected the gradient increases as the thickness of the Al sample increases.

The gradient ( $\mathrm{P} / \delta$ - or stiffness constant) values for these plots, can be used to determine an effective Young's modulus value to both verify the measurement method (for Al-Si samples with known stiffness) and to use as input data for the FEA of sandwich structures. To do this requires some consideration of the mechanics of beams in bending. The elastic deflection, $\delta$, for a slender beam, under 3-point loading, can be described by equation 1, (the so-called Euler-Bernoulli 
equation) where $\mathrm{P}$ is the load applied, $\mathrm{L}$ the span, $\mathrm{E}$ the Young's modulus and I, the second moment of area of the beam.

$$
\delta=\frac{P L^{3}}{48 E I}
$$

For thicker beams, shear stresses in the section cannot be ignored and a second term to capture this is added. Equation 2 (the so-called Timoshenko equation) presents this case, where $A$ is the cross sectional area, $\mathrm{G}$ is the shear modulus, which, for a Poisson's ratio of 0.33 , can be approximated to $3 \mathrm{E} / 8$ and $\mathrm{K}$ is the shear coefficient (often accepted to be $5 / 6$ for rectangular beams [19]). The ISO 178 standard [20], for beams with a span of $64 \mathrm{~mm}$, uses equation 1 to determine the beam bending behaviour, for a beam thickness not exceeding $4 \mathrm{~mm}$. Equation 3 shows the result of rearranging equation 2 , using the simplification for the shear modulus stated earlier, to enable the Young's modulus of thick beams to be determined.

$$
\begin{aligned}
& \delta=\frac{P L^{3}}{48 E I}+\frac{P L}{4 A G K} \\
& E=\frac{P}{\delta} \frac{A K L^{3}+32 I L}{48 A I K}
\end{aligned}
$$

A comparison of $\mathrm{P} / \delta$, determined from equations 1 and $2, \mathrm{FEA}$ and experimental data, is shown in Figure 5. It is evident that FEA simulations follow the thick beam model, and both are in good agreement with experimental measurements. Although the fit to the other data looks good for the thin beam model, for thin beams, the discrepancy increases with increasing beam thickness. For example, for a $16 \mathrm{~mm}$ thick beam the stiffness $(\mathrm{P} / \delta)$ predicted by the thin beam model is $20 \%$ higher than those for both the thick beam model and FEA. For the thick beams investigated in this work, and the simplifications listed above, the contribution from the shear term in equation 2 is no longer insignificant. For the $4 \mathrm{~mm}$ thick ISO 178 standard [20], the shear contribution is $1 / 80^{\text {th }}$ that for bending, for $16 \mathrm{~mm}$ thick samples it is $1 / 5^{\text {th }}$ (accounting for the $20 \%$ difference 
observed). Using the thick beam model, the average Young's modulus calculated from experimental data for the Al beam was $70.2 \pm 0.4 \mathrm{GPa}$, close to that expected for the LM6 alloy used (typically $71 \mathrm{GPa}$ [17]). Close matching gave confidence to the testing methodology and the value measured was used as the input for modelling. Calculations using equation 1 yielded an average Young's modulus of $67.4 \pm 1.5 \mathrm{GPa}$, indicating that ignoring the contribution from the shear term for thick beams can lead to significant under-prediction of the Young's modulus. Subsequent FE modelling of sandwich structures also requires an input value for the Young's modulus of the syntactic foam, which is treated effectively as a continuous material. $\mathrm{P} / \delta$ values were measured for syntactic foams in the range of roughly 2700 to $2900 \mathrm{~N} / \mathrm{mm}$, for samples with densities in the approximate range of 1.13 to $1.15 \mathrm{~g} / \mathrm{cc}$. Again the repeatability in the measurements over the 4 loading cycles was high. Figure 6 plots the Young's modulus, calculated using $\mathrm{P} / \delta$ values and equation 3 , against the density. Although there is some scatter, the Young's modulus increases with density, following a power law dependence with exponent close to 2 , in keeping with the behaviour for more conventional metal foams and porous metals $[1,6]$. The mean density, $1.14 \mathrm{~g} / \mathrm{cc}( \pm 0.006)$, corresponds to a Young's modulus of approximately $7000 \mathrm{MPa}$ (7007 \pm 72 ) and is similar to the stiffness measured in compression for syntactic metal foams containing these same EGP [8].

Since the thick beam equation has been shown to be an accurate predictor of the stiffness of simple beams, it was used, in conjunction with the mean Young's modulus and density determined from syntactic metal foam (SMF) data presented in Figure 6, to compare the stiffness $(\mathrm{P} / \delta)$ and mass for $\mathrm{Al}$ and syntactic metal foam beams. This comparison is presented in Figure 7, over a range of practical thicknesses, given that the EGP are $>2 \mathrm{~mm}$ in diameter. The plot reveals an intersection at a stiffness of roughly $28000 \mathrm{~N} / \mathrm{mm}$, above which mass savings for SMFs are no longer achievable. Below this point, mass savings are typically no more than $7 \%$. Despite the 
shear coefficient, $K$, not being a constant over the range of beam thicknesses investigated [19], the predicted effect on $\mathrm{P} / \delta$ is small for the materials and geometries presented herein.

For thin beams with very low stiffness (less than $500 \mathrm{~N} / \mathrm{mm}$ ), both materials will behave like "Euler" beams and their masses (for a given deflection) will be proportional to $\rho / E^{1 / 3}$. Using values measured in this study, SMF beams could offer a 9\% mass saving over Al, under these rather limited conditions. To achieve a stiffness of roughly $30000 \mathrm{~N} / \mathrm{mm}$, the $10 \mathrm{~mm}$ thick Al beam required still behaves much like an Euler beam (see Figure 5). A SMF beam with the same stiffness must be much thicker and, since the ratio of the shear to bending term in equation 2 increases with $\mathrm{d}^{2}$, it will be much less efficient at resisting bending. For this reason, the mass savings for the thick syntactic metal foam beams diminishes with increasing stiffness, to a point where mass savings are no longer possible.

\section{Sandwich structures}

In order to ensure consistency in testing and modelling, the preforms used to make sandwich structures were screened, by mass, to ensure the resulting cores would lie within same the density range as was tested previously $(1.14 \pm 0.01 \mathrm{~g} / \mathrm{cc})$. Table 1 presents the expected masses for sandwich structures based on a core thickness of $10 \mathrm{~mm}$ and a core density of $1.14 \mathrm{~g} / \mathrm{cc}$ and compares it with the samples tested. The close agreement indicates that target densities were achieved.

Figure 8 and Table 1 compare experimental data for the elastic deflection of sandwich beam structures with those predicted using FEA. Despite the small displacements measured, the experimental plots show good linearity and the standard deviation for the 4 testing cycles was less than $2 \%$. As expected, sandwiches with thicker face sheets show a stiffer response. FEA P/ $\delta$ plots are also presented, given by dotted lines, modelled using a Young's modulus for the core of $7000 \mathrm{MPa}$. Agreement in this figure, and with data in Table 1, is very close, supporting the validity of assuming "perfect" bonding between the faces and the core in these novel sandwich 
structures. It is worth noting that substitution with Young's modulus data at the extremes of the range measured for the core, presented in Figure 6 , resulted in $\mathrm{P} / \delta$ predictions that varied by less than $0.5 \%$ from those calculated using the mean value, well within the $2 \%$ scatter in experimental measurement.

For sandwich structures, an equation with a similar form to the thick beam equation (equation 2) is often used to predict the elastic deflection [21]. Equation 4 defines the deflection for a sandwich structure where;

$$
\delta=\frac{P L^{3}}{48 E I}+\frac{P L}{4 A G}
$$

the equivalent shear rigidity is;

$$
(A G)=\frac{G_{c} b d^{2}}{c}
$$

the equivalent flexural rigidity is;

$$
(E I)=\frac{E_{f} b t^{3}}{6}+\frac{E_{c} b c^{3}}{12}+\frac{E_{f} b t d^{2}}{2}
$$

and where $\mathrm{c}$ and $\mathrm{t}$ are the thickness of the core and faces and $\mathrm{d}$ the total thickness of the sandwich structure. Subscripts $f$ and $c$ denote the properties in the face and core respectively.

Table 1 shows that predictions using the analytical model differ greatly from experimental measurements and FE modelling, and that the difference increases with increasing face sheet thickness. Figure 9 plots the predicted bending response for FEA and the analytical model, where good agreement is only observed for core to face sheet thickness ratio of 10:1 (i.e. $1 \mathrm{~mm}$ face sheets on a $10 \mathrm{~mm}$ thick core), supporting a similar good fit in [1] for a ratio of 12.5:1; and in [10] for very thin composite face sheets. It is clear from this study that, as the faces become thicker, and shear in the face sheets becomes increasingly significant, the analytical model significantly overestimates the stiffness of the beam. 
Since FE simulations most accurately predict the elastic deflection response of these thick sandwich structures, Figure 10 plots curves generated from $\mathrm{FE}(\mathrm{P} / \delta)$ data for sandwich structures with increasing core thickness and various face sheet thicknesses, and $\mathrm{Al}$, enabling a comparison of the masses (in this instance for the material between the lower supports) for beams with the same stiffness. Experimental data points from Table 1 (but with the mass corrected for that between the supports) are overlaid on this plot and fit well to positions corresponding to $10 \mathrm{~mm}$ thick cores, on curves matching their respective face thicknesses. Mass savings are not possible for any of the core thicknesses with $5 \mathrm{~mm}$ thick face sheets. For $4 \mathrm{~mm}$ faces, mass savings are very small and only achieved for a stiffness below approximately $50000 \mathrm{~N} / \mathrm{mm}$. These results illustrate diminishing structural efficiency as the Al face sheets increase in thickness, a result of increasing levels of shear occurring within them.

By approximating the curves in Figure 10 to, in the case of the $\mathrm{Al}$, a power law, and for the SMF sandwiches, a $3^{\text {rd }}$ order polynomial (all with $\mathrm{R}^{2}$ fits $>0.999$ ) the difference in mass at any given target stiffness was calculated, and is plotted in Figure 11. Maximum mass savings are 31, 21 and $11 \%$ for 1,2 and $3 \mathrm{~mm}$ face sheets respectively, with the stiffness and core thickness at these maxima increasing with increasing face sheet thickness. As was the case for the monolithic beams, the thick cores needed to attain high stiffness are less efficient at resisting bending and mass savings are limited to stiffnesses below 62000,69000 and $66000 \mathrm{~N} / \mathrm{mm}$ for 1,2 and $3 \mathrm{~mm}$ faces respectively. The mass savings for these composite sandwich structures, and the range over which savings can be achieved, do, however, greatly exceed those for the core material alone, illustrating the well-known benefits of using sandwich structures to reduce mass.

\section{Summary}

This study demonstrates that the uniqueness of the manufacturing process used is both its advantage, the face sheets are integrally bonded to the core, and its limitation, the face sheets 
are necessarily thick, owing to the need for metal to flow around the core "insert". Altering the casting process or adopting a machining step, to reduce the face thickness, will clearly benefit the performance and increase mass savings, but further research is required to determine if this can be achieved without adding costs that might not be justified for some applications.

The incremental development of the understanding of the material performance, and how to model it, has shown that the structural characteristics of these materials warrant FE-based modelling of the elastic deformation behaviour, but that the methodology is simple and accurate. A reliance on predictions from established analytical models, which have proven themselves accurate for foam sandwiches with thin face sheets, will lead to significant over-estimations of the stiffness and thus the mass savings that might be possible for these sandwich structures.

Modelling (supported by one experimental datum point) shows that mass savings between 20 and $30 \%$ are achievable for these novel structures, but are dependent upon the stiffness $(\mathrm{P} / \delta)$ required. At very high stiffness (> $60000 \mathrm{~N} / \mathrm{mm}$ ) mass savings, compared with solid Al structures, are not possible for the system investigated in this work. The simple, yet accurate, FE modelling presented, makes a significant contribution to aiding the design of syntactic metal foam structures and could be applied to more complex geometries. Additionally, it could offer insights into how further mass savings might be achieved, for example if the core density and stiffness were altered.

\section{Acknowledgements}

The authors would like to acknowledge Peter Hanratty for his support with the mechanical testing. This research did not receive any specific grant from funding agencies in the public, commercial, or not-for-profit sectors.

\section{Data Availability}


These data are being used in ongoing work but some will be made available on request.

\section{References}

1. M.F. Ashby et al. Metal Foams: A Design Guide. Burlington, Butterworth-Heinemann, 2000.

2. García-Moreno F. Commercial Applications of Metal Foams: Their Properties and Production. Materials 2016, 9, 85; doi:10.3390/ma9020085

3. Banhart J, Baumeister J. Deformation characteristics of metal foams. Journal of Materials Science 1998 (33) 1431-1440.

4. Neugebauer B, Lies R, Hohlfeld J, Hipke T. Adhesion in sandwiches with aluminum foam core, Production Engineering 2007 (1) 271-278.

5. Banhart J, Seeliger H. Aluminium Foam Sandwich Panels: Manufacture, Metallurgy and Applications. Advanced Engineering Materials. 2008 (9) 793-802.

6. Banhart J. Manufacture, characterisation and application of cellular metals and metal foams, Progress in Materials Science 2001 (46) 559-632.

7. Banhart J. Aluminum Foams: On the Road to Real Applications. MRS Bulletin. 2003 (28) 290295.

8. Wright A, Kennedy A. The Processing and Properties of Syntactic Al Foams Containing Low Cost Expanded Glass Particles. Advanced Engineering Materials. 2017 (19): doi:10.1002/adem.201600467.

9. Omar MY, Xiang C, Gupta N, Strbik III OM, Cho K. Syntactic foam core metal matrix sandwich composite: Compressive properties and strain rate effects. Materials Science \& Engineering. 2015 (A643) 156-168.

10. Omar MY, Xiang C, Gupta N, Strbik III OM, Cho K. Syntactic foam core metal matrix sandwich composite under bending conditions. Materials and Design. 2015 (86) 536-544. 
11. Styles $\mathrm{M}$, Compston $\mathrm{P}$, Kalyanasundaram $\mathrm{S}$. Finite element modelling of core thickness effects in aluminium foam/composite sandwich structures under flexural loading. Composite Structures. 2008 (86) 227-232.

12. Styles $M$, Compston $P$, Kalyanasundaram $\mathrm{S}$. The effect of core thickness on the flexural behaviour of aluminium foam sandwich structures. Composite Structures. 2007 (4) 532-538.

13. Potluri R, Kumar AE, Rajuc MN, Prakash Babu KR. Finite Element Analysis of Cellular Foam Core Sandwich Structures. Materials Today. 2017 (4) 2501-2510.

14. Betts $\mathrm{C}$. Benefits of metal foams and developments in modelling techniques to assess their materials behaviour: a review, Materials Science and Technology, 2012 (28) 129-143.

15. Sha JB, Yip TH, Teo MH. FEM modelling of single-core sandwich and 2-core multilayer beams containing foam aluminum core and metallic face sheets under monolithic bending, Progress in Natural Science, Materials International. 2011 (21) 127-138.

16. Omya, "Product Information: Glass Ceramics, EGP40 [Technical Data]," private communication with supplier, 2015.

17. Product data LM6 alloy, Norton Aluminium, http://www.nortal.co.uk/LM6/ accessed 29/11/2019.

18. Langston P, Kennedy A.R. Discrete element modelling of the packing of spheres and its application to the structure of porous metals made by infiltration of packed beds of $\mathrm{NaCl}$ beads. Powder Technology, 2014 (268) 210-218.

19. Gruttmann F, Wagner W. Shear correction factors in Timoshenko's beam theory for arbitrary shaped cross-sections. Computational Mechanics. 2001 (27) 199-207.

20. ISO 178, https://www.iso.org/obp/ui/\#iso:std:iso:178:ed-6:v1:en, [Accessed 310 2019].

21. Allen H.G. Theory of sandwich beams and plates (volume 1: fundamental aspects), in: Shenoi RA, Wellicome JF, editors, Composite Materials in Maritime Structures, NY, Cambridge University Press, 1993. p 205-235. 


\section{Figure captions}

Figure 1 Images of (a) EGP and (b) an EGP preform located within the metal casting mould used to create sandwich structures

Figure 2 Images showing (a) as-cast sandwich components and (b) a machined sandwich component with a $10 \mathrm{~mm} \mathrm{SMF} \mathrm{core} \mathrm{and} 2 \mathrm{~mm}$ Al-Si face sheets

Figure 3 Schematic illustrations for both the testing method and the FEA models for 3 point bending of (a) the syntactic foam sample and (b) the sandwich structure.

Figure 4 Load-displacement plots for $28 \mathrm{~mm}$ wide Al and syntactic metal foam (SMF) samples of different thickness

Figure 5 Gradient of load-displacement (stiffness) as a function of the thickness of Al beams, calculated using different models and compared with experimental measurements

Figure 6 Plot of Young's modulus (calculated using equation 3) as a function of density for syntactic metal foam samples

Figure 7 Comparison of mass, as a function of $\mathrm{P} / \delta$, for $\mathrm{Al}$ and syntactic metal foam samples (using equation 2)

Figure 8 Force - displacement plots for sandwich structures, compared with FEA predictions (dotted lines)

Figure 9 Comparison of FEA and analytical model (core $=10 \mathrm{~mm}$ ) for $\mathrm{P} / \delta$ as a function of skin thickness

Figure 10 Beam mass (between the lower supports) as a function of $\mathrm{P} / \delta$ for $\mathrm{Al}$ and syntactic metal foam sandwich structures (experimental data are shown as single points)

Figure 11 Mass differences for SMF sandwich structures compared to Al beams with the same stiffness. 
Tables

Table 1 Comparison of $\mathrm{P} / \delta$ (in $\mathrm{N} / \mathrm{mm}$ ) and mass (in $\mathrm{g}$ ) for sandwich structures with a core thickness of $10 \mathrm{~mm}$ and different face sheet thickness, (\% of the experimental value is shown in brackets)

\begin{tabular}{cccccc}
$\begin{array}{c}\text { Face sheet } \\
\text { thickness }\end{array}$ & $\begin{array}{c}\mathrm{P} / \delta \\
\text { Experimental }\end{array}$ & $\mathrm{P} / \delta$ FEA & $\mathrm{P} / \delta$ Analytical & $\begin{array}{c}\text { Theoretical } \\
\text { mass }\end{array}$ & $\begin{array}{c}\text { Actual } \\
\text { mass }\end{array}$ \\
\hline $2 \mathrm{~mm}$ & $20424 \pm 309$ & $20237(99 \%)$ & $26577(130 \%)$ & 35.5 & 35.6 \\
$3 \mathrm{~mm}$ & $28866 \pm 411$ & $28137(97 \%)$ & $41466(144 \%)$ & 44.2 & 44.2 \\
$4 \mathrm{~mm}$ & $38399 \pm 755$ & $37230(97 \%)$ & $58357(152 \%)$ & 52.8 & 53.0 \\
$5 \mathrm{~mm}$ & $48357 \pm 865$ & $47792(99 \%)$ & $77320(160 \%)$ & 61.4 & 61.5
\end{tabular}

